II presente documento viene fornito attraverso il servizio NILDE dalla Biblioteca fornitrice, nel rispetto della vigente normativa sul Diritto d'Autore (Legge n.633 del 22/4/1941 e successive modifiche e integrazioni) e delle clausole contrattuali in essere con il titolare dei diritti di proprietà intellettuale.

La Biblioteca fornitrice garantisce di aver effettuato copia del presente documento assolvendo direttamente ogni e qualsiasi onere correlato alla realizzazione di detta copia.

La Biblioteca richiedente garantisce che il documento richiesto è destinato ad un suo utente, che ne farà uso esclusivamente personale per scopi di studio o di ricerca, ed è tenuta ad informare adeguatamente i propri utenti circa i limiti di utilizzazione dei documenti forniti mediante il servizio NILDE.

La Biblioteca richiedente è tenuta al rispetto della vigente normativa sul Diritto d'Autore e in particolare, ma non solo, a consegnare al richiedente un'unica copia cartacea del presente documento, distruggendo ogni eventuale copia digitale ricevuta.

Biblioteca richiedente: Biblioteca Dipartimento Scienze di base e applicate per l'ingegneria - Sez.Fisica

Data richiesta:

05/11/2020 16:13:45

Biblioteca fornitrice: Biblioteca INAF-Osservatorio Astrofisico di Catania e Dipartimento di Fisica e Astronomia

Data evasione: $\quad$ 05/11/2020 16:29:25

Titolo rivista/libro: $\quad$ Proceedings of SPIE

Titolo articolo/sezione: The SPARC_LAB femtosecond synchronization for electron and photon pulsed beams

Autore/i: $\quad$ Bellaveglia,Gallo, Piersanti

ISSN:

1996-756X

DOI:

Anno:

Volume:

9512

Fascicolo:

Editore:

Pag. iniziale:

Pag. finale: 


\section{The SPARC LAB femtosecond synchronization for electron and photon pulsed beams}

Bellaveglia, M., Gallo, A., Piersanti, L., Pompili, R., Gatti, G., et al.

M. Bellaveglia, A. Gallo, L. Piersanti, R. Pompili, G. Gatti, M. P. Anania, M. Petrarca, F. Villa, E. Chiadroni, A. Biagioni, A. Mostacci, "The SPARC LAB femtosecond synchronization for electron and photon pulsed beams," Proc. SPIE 9512, Advances in X-ray Free-Electron Lasers Instrumentation III, 95120V (12 May 2015); doi: 10.1117/12.2185103

SPIE. Event: SPIE Optics + Optoelectronics, 2015, Prague, Czech Republic 


\title{
The SPARC_LAB femtosecond synchronization for electron and photon pulsed beams
}

\author{
M. Bellaveglia $a$, M. P. Anania ${ }^{a}$, A. Biagioni ${ }^{a}$, E. Chiadroni $a$, A. Gallo ${ }^{a}$, G. Gatti $^{a}$, A.

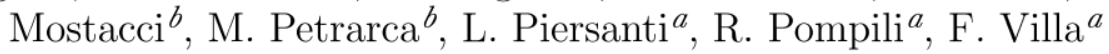 \\ ${ }^{a}$ INFN-LNF, Via Enrico Fermi 40, 00044 Frascati (Rome), Italy \\ ${ }^{b}$ Universita' degli studi di Roma La Sapienza, P.le Aldo Moro 5, 00185 Roma (Italy)
}

\begin{abstract}
The SPARC_LAB complex hosts a $150 \mathrm{MeV}$ electron photo-injector equipped with an undulator for FEL production (SPARC) together with a high power TW laser (FLAME). Recently the synchronization system reached the performance of $<100 \mathrm{fs}_{R M S}$ relative jitter between lasers, electron beam and RF accelerating fields. This matches the requirements for next future experiments: (i) the production of X-rays by means of Thomson scattering (first collisions achieved in 2014) and (ii) the particle driven PWFA experiment by means of multiple electron bunches. We report about the measurements taken during the machine operation using BAMs (Bunch Arrival Monitors) and EOS (Electro-Optical Sampling) system. A new R\&D activity concerning the LWFA using the external injection of electron bunches in a plasma generated by the FLAME laser pulse is under design. The upgrade of the synchronization system is under way to guarantee the $<30$ fs RMS jitter required specification. It foresees the transition from electrical to optical architecture that mainly affects the reference signal distribution and the time of arrival detection performances. The new system architecture is presented together with the related experimental data.
\end{abstract}

Keywords: synchronization, PLL, linac, FEL, plasma acceleration, Thomson scattering

\section{INTRODUCTION}

This paper describes the status of the synchronization system implemented at the SPARC_LAB complex in Frascati National Laboratories of INFN. ${ }^{1}$ The SPARC LAB R\&D facility hosts a $150 \mathrm{MeV}$ electron photoinjector equipped with an undulator for FEL production (SPARC) together with a high power TW laser (FLAME). Some future experiments such as (i) the production of X-rays by means of Thomson scattering (first collisions achieved in 2014), ${ }^{2}$ (ii) the particle driven PWFA experiment by means of multiple electron bunches $^{3}$ and (iii) the LWFA using the external injection of a SPARC electron bunch in a plasma generated by the FLAME laser pulse ${ }^{4}$ will be performed in the next future. The requested performance of the synchronization system is different for each of the experiments. This is due to the different electron and photon beam time duration, that varies from some ps to tens of fs, and, if present, to the different beam interaction schemes (co-propagating, head-on). In particular the requested relative time jitter between the sub-system of the facility (namely $\mathrm{S}$ and C-band RF systems and two laser systems), varies from $<1 \mathrm{ps} R M S$ for the Thomson experiment down to $<30 \mathrm{fs}_{R M S}$ for the LWFA with external injection experiment.

\subsection{General concepts about synchronization}

A general architecture for a synchronization system of a "linac + experiment" complex is shown in figure 1. Each sub-system can be viewed as a free running optical or electrical oscillator. In the facility is present one stable reference oscillator whose signal is distributed along the machine to the sub-system clients. The reference oscillator can generate either an electrical signal, that is distributed to the clients with coaxial cables, or an optical signal, that is distributed by means of optical fibers. The latter case is suitable for systems with higher performance because of the higher achievable resolution in the time jitter measurements and the possibility of transporting short reference pulses with moderate signal attenuation over long distances. The synchronization between subsystems is then basically obtained locking the frequency of each client

Further author information: marco.bellaveglia@lnf.infn.it

Advances in X-ray Free-Electron Lasers Instrumentation III, edited by Sandra G. Biedron Proc. of SPIE Vol. 9512, 95120V · @ 2015 SPIE · CCC code: 0277-786X/15/\$18 doi: $10.1117 / 12.2185103$

Proc. of SPIE Vol. $951295120 \mathrm{~V}-1$ 


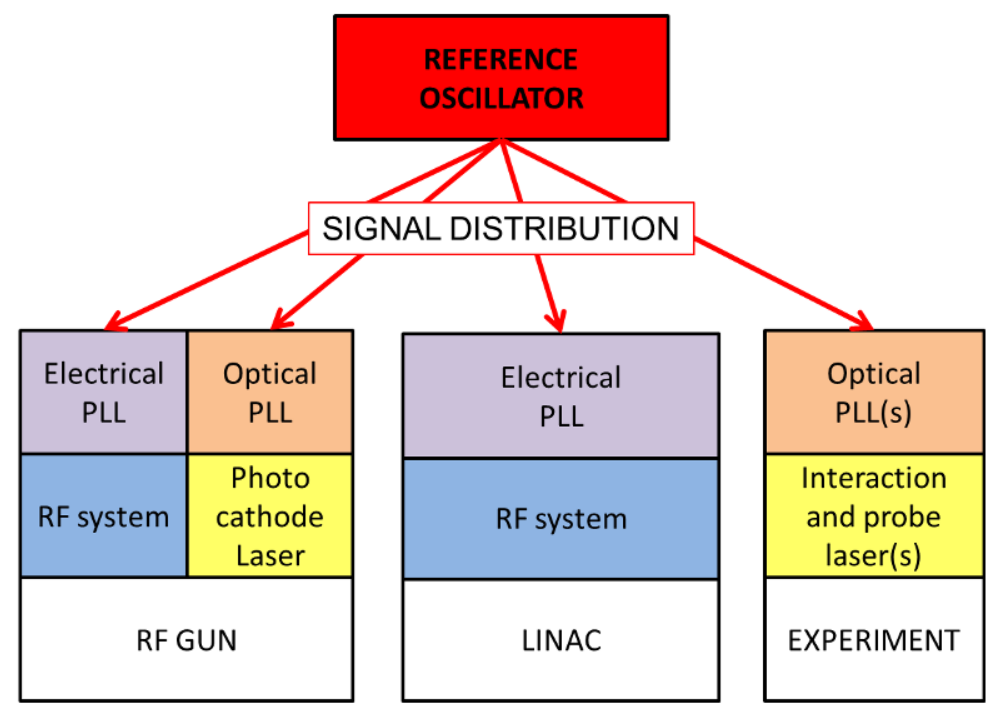

Figure 1. Linac synchronization system general architecture.

oscillator to that of the reference by means of phase locked loops (PLLs). Different clients require different approaches so that the PLL can be composed by electrical, optical and/or mechanical devices. The aim of such a system is to minimize the relative time jitter among the clients, conforming as much as possible the absolute jitter to that of the refence oscillator. In the next chapters we will examine the various cases, reporting the results achieved at SPARC_LAB concerning the various sub-systems and the measurements on the real beams during the experimental shifts.

\section{THE PRESENT SPARC_LAB SYNCHRONIZATION SYSTEM}

The synchronization is presently provided by an analog RF reference signal distributed through a coaxial cable network, and client lock-in is based on electronic PLL implementing $\mu$-wave techniques. The layout of the system is shown in figure 2. In the near future we plan to perform much more demanding experiments, such as external injection of very short electron bunches in a laser-driven plasma wave for post acceleration. The synchronization request is dramatically stringent in this case, reaching the level of $<30 \mathrm{fs}$, which requires a technological leap to optical techniques. To reach this new frontier we are preparing the migration of the SPARC_LAB synchronization system from electrical, cable based distribution and lock-in, to full optical, fiber based, architecture as described in section 3 .

\subsection{Reference oscillator}

The present SPARC_LAB Reference Master Oscillator (RMO) is an RF crystal oscillator providing a phase coherent signal at the various RF frequencies used in the facility, namely $2856 \mathrm{MHz}, 5712 \mathrm{MHz}, 2142 \mathrm{MHz}$. It is a custom product with low phase noise profile. A measurement of the phase noise power spectrum at $2856 \mathrm{MHz}$ together with the integrated absolute time jitter is reported in figure 4 .

\subsection{Laser system synchronization}

\subsubsection{Lock of the FLAME interaction laser}

The SPARC_Lab facility integrates the SPARC linac and the FLAME interaction laser capable to deliver $10 \mathrm{~Hz}$ infra-red (TiSa, $800 \mathrm{~nm}$ ) pulses with energies $>1 \mathrm{~J}$ and durations $<30 \mathrm{fs}$, i.e. in the $100 \mathrm{TW}$ peak power regime. Such a laser is also a scientific instrument for standalone experiments, and it is used in combination with the linac for experiments of electron-photon interaction. Recently, the first experiment of this category has been performed, which has shown the evidence of production of X-rays by electron-photon Thomson backscattering. The laser is synchronized at the optical oscillator level by means of a standard 


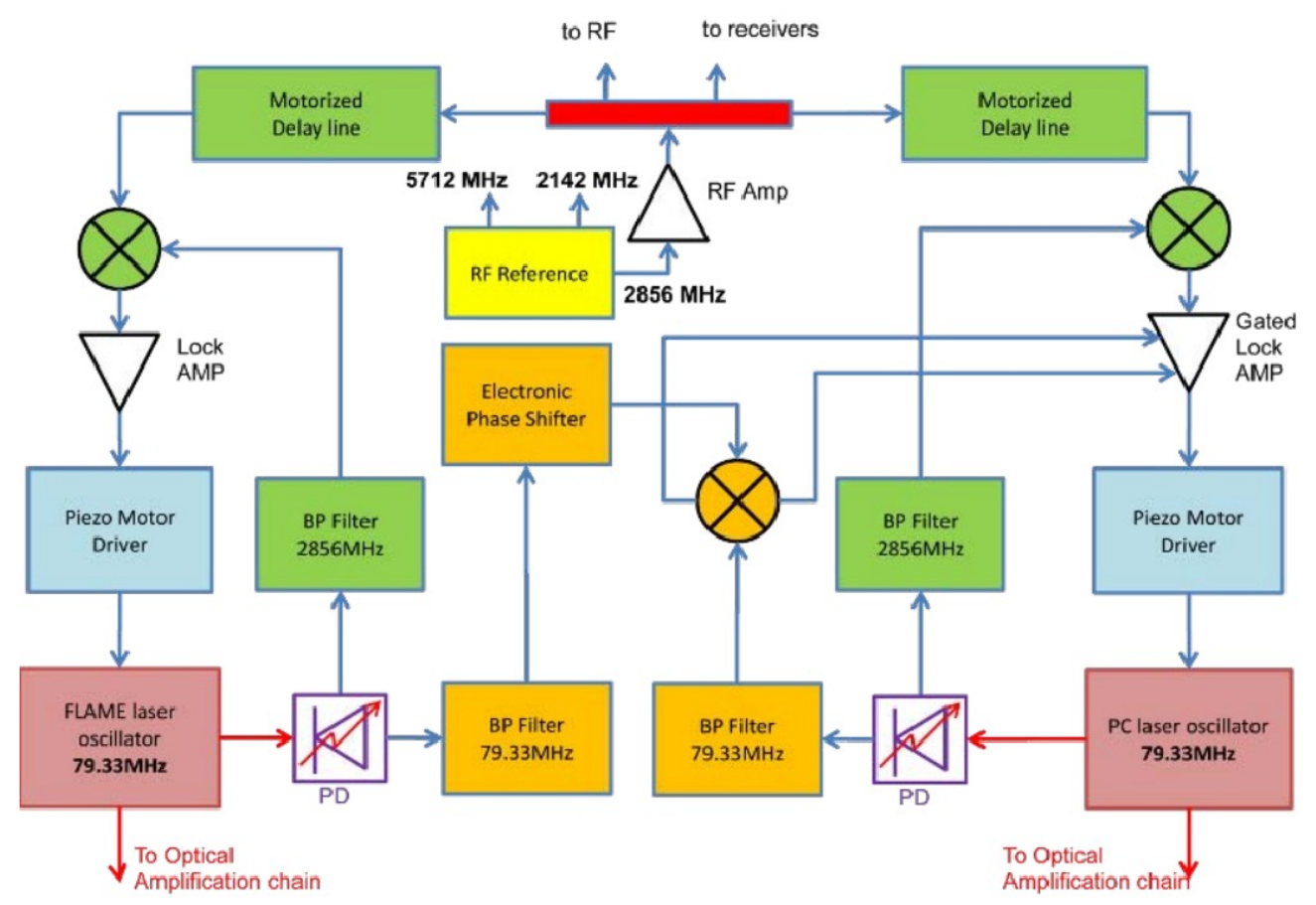

Figure 2. Layout of the present SPARC_LAB synchronization system

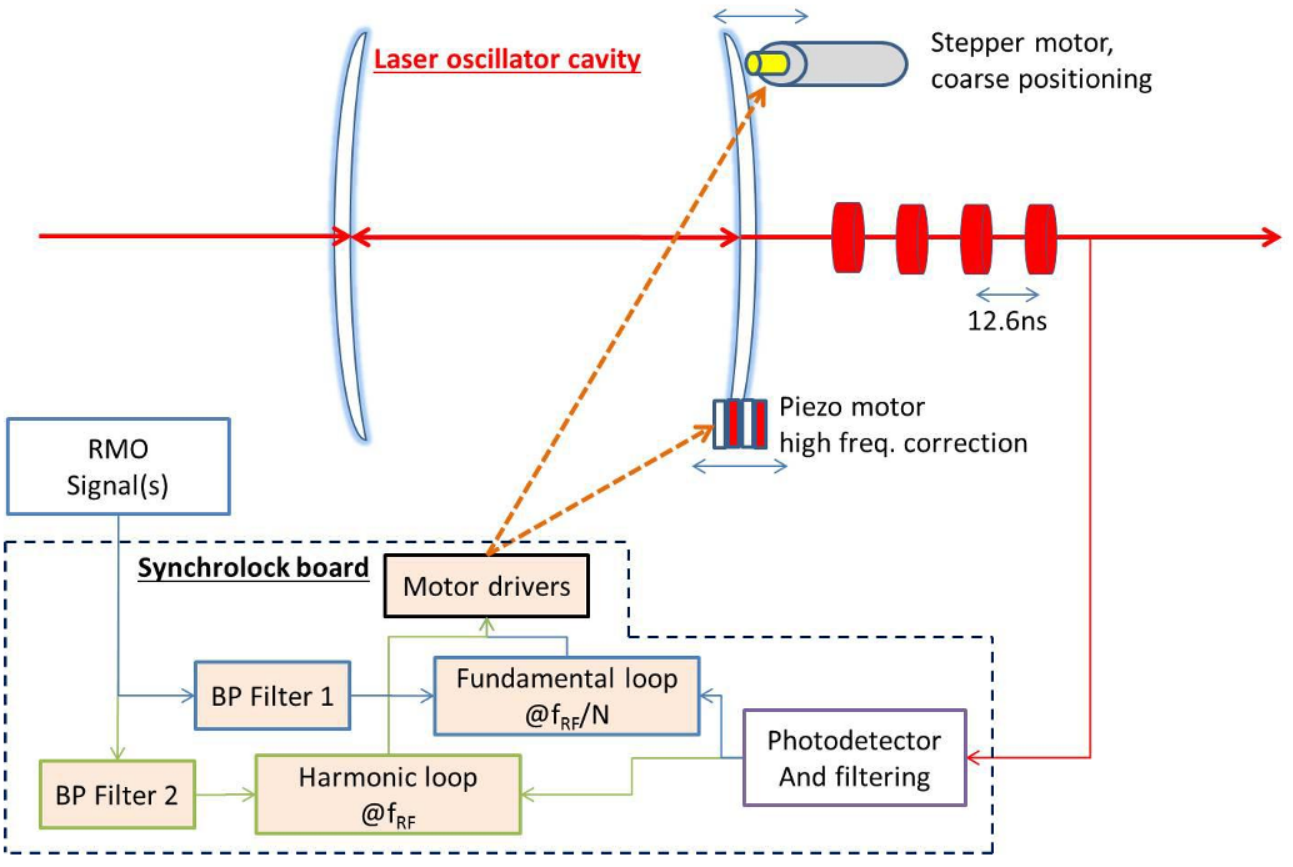

Figure 3. Schematics of a standard laser client synchronization technique

locking technique. The oscillator frequency is $R F / 36=79+1 / 3 \mathrm{MHz}$ and it is locked to the RF reference by means of an electro-mechanical PLL shown in figure 3. The length of the optical cavity of the laser oscillator is adjusted by a piezo-controller moving a mirror, so that the laser oscillator behaves like a VCO (Voltage Controlled Oscillator). A sample of the oscillator pulse train illuminates a photodetector with adequate bandwidth, and the required harmonics $(n=36, f=2856 \mathrm{MHz})$ of the signal is extracted with a bandpass filter. The signal is then phase compared to the reference, and the error signal is treated with a 
low frequency amplifier to shape the loop transfer function and finally connected to the piezo-motor driver to lock the phase and the frequency of the optical cavity. A stepper motor provides a coarse mirror positioning, in order to operate the piezo-motor at the center of its dynamic range. In the FLAME case all this hardware is embedded in the control electronics provided by the manufacturer. The measured residual SSB phase noise of the FLAME oscillator is shown in figure 4 and it corresponds to an integrated absolute jitter of $87 \mathrm{fs}_{R M S}$ in the $10 \mathrm{~Hz} \div 10 \mathrm{MHz}$ band. The repetition rate of the high energy laser pulses is $10 \mathrm{~Hz}$, which means that one oscillator pulse every $100 \mathrm{~ms}$ is selected and amplified. All the oscillator pulses, including the selected and amplified ones, are synchronized to the reference. The loop phase detector works at the $2856 \mathrm{MHz}$, the 36 th harmonics of the laser working frequency. In general high frequency phase detection is more accurate and leads to better PLL performances. However, when locking a low-frequency oscillator with a PLL working on its Nth harmonics, there are N possible final phase states of the oscillator. This uncertainty may affect the facility synchronization whenever more lasers are locked to a common harmonic reference. We chose to prevent such a problem putting extra conditions to the lock of the SPARC photocathode laser (see paragraph $2.2 .3)$.

\subsubsection{Lock of the photocathode laser}

The SPARC linac beam originates in an RF gun cavity, where the electrons are extracted from a metallic photocathode by an UV energetic laser pulse at repetition rate of $10 \mathrm{~Hz}$. The PC (Photo Cathode) laser defines the launching time of the beam, and together with the RF fields which control the capture processes, defines the time of arrival of the bunch at the end of the linac. The PC laser synchronization to the reference is made exactly in the same way already described for the FLAME laser. The PC laser oscillator (MIRA model produced by Coherent Inc.) has the same repetition rate as the FLAME one $(79+1 / 3 \mathrm{MHz})$ and was originally equipped with its own synchronization hardware and software. The laser has been operated with its original control electronics for few years, with a measured jitter in the $200 \div 300$ fs range. The phase detection electronics and the error signal amplifier of the PC laser PLL has been replaced to improve its performance. The PLL front end has been redesigned to operate at the main SPARC RF frequency (2856 MHz). A new photodiode with $>3 \mathrm{GHz}$ bandwidth has been installed to monitor the phase of the MIRA pulse train. A dedicated error amplifier based on OP AMPs has been designed, built and tested. In particular, we found very effective to increase the multiplicity of the PLL integrator. ${ }^{5}$ The MIRA integrated absolute time jitter is reduced to $\approx 70 \mathrm{fs}$, dominated by the intrinsic noise of the reference. The SSB power spectrum of the phase is shown in figure 4. Further developments are under design to compensate also the piezo actuator resonance at $50 \mathrm{kHz}$ that affects the closed-loop transfer function of the system at lower frequencies (limiting the bandwidth of the response to few $\mathrm{kHz}$ ). ${ }^{6}$

\subsubsection{FLAME and PC laser low frequency lock}

To achieve a good sensitivity in the phase detection, both the laser PLLs work at the main linac frequency $2856 \mathrm{MHz}$. Then, every $100 \mathrm{~ms}$, only a pulse of the $79+1 / 3 \mathrm{MHz}(R F / 36)$ train is amplified. The selection of the pulse is made using a HV electro-optical modulator (typically a Pockel's cell) driven by the $10 \mathrm{~Hz}$ machine trigger, conveniently delayed. If the two lasers locking systems are left independent, the relative delay can change every time they are locked, since the synchronization is made at the 36th harmonic of the repetition rate and each laser can be locked in 36 positions within one period of the fundamental frequency, i.e. where the error signal is zeroed. To avoid this, as shown in figure 2, the error amplifier OP AMPs are also used to gate the MIRA PLL, i.e. to condition the MIRA lock to the actual FLAME pulse temporal position to avoid the uncertainty arising from the harmonic PLL. In practice, the relative phase between MIRA and FLAME is measured at the laser oscillators fundamental frequency $79+1 / 3 \mathrm{MHz}$, and the OP AMPs of the MIRA PLL are enabled only if the measured phase is within the $\pm 5^{\circ}$ range (i.e. $1 / 36$ of the entire $360^{\circ}$ range). By doing this the MIRA lock is aligned with FLAME and the uncertainty is removed. The coarse (12.6 ns step) relative shift between the 2 lasers is controlled by a phase shifter inserted in the FLAME channel upstream the $79+1 / 3 \mathrm{MHz}$ phase detector. The fine temporal shift between the 2 lasers is controlled by two motorized delay lines (trombones) moving the phase of the $2856 \mathrm{MHz}$ PLL reference. 


\subsection{RF system synchronization}

Since the system architecture uses a reference crystal oscillator, its RF output is directly used to seed the $\mathrm{RF}$ power plants. The oscillator is designed to provide many coherent frequencies and is able to provide the signals to drive the C-band $(5712 \mathrm{MHz})$ and S-band $(2856 \mathrm{MHz})$ klystrons. The RF S-band power stations (modulator + klystron) provide the RF accelerating field pulse with duration of $1 \mu$ s for the RF line 1 (feeding RF gun, $3^{\text {rd }}$ accelerating section and RF deflector) and $4.5 \mu$ s for the RF line 2 (feeding the $1^{\text {st }}$ and $2^{\text {nd }}$ accelerating section through a pulse compression system). For a detailed description of the Low Level RF (LLRF) system see. ${ }^{8}$

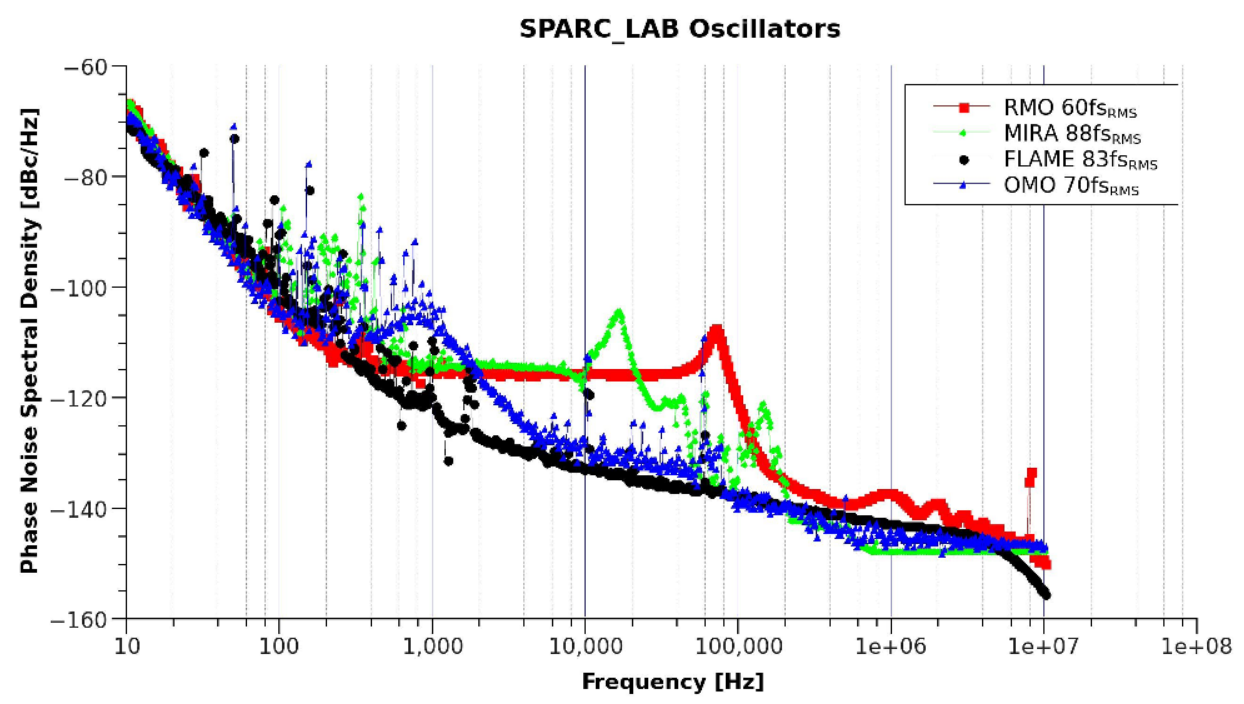

Figure 4. Phase noise spectral density and integrated jitter of the SPARC_LAB oscillators

\subsubsection{Klystron stabilization loop}

The architecture of the S-band modulators is based on a classical PFN (Pulse Froming Network) with capacitor array and a thyratron as pulse switch. This design adds a small shot-to-shot phase uncertainty in the RF pulse, so each of the S-band power station as provided by the manufacturer do not preserve the good stability of the driving signal. To overcome the problem, we designed an intra-pulse PLL with large $(\approx 1 \mathrm{MHz})$ bandwidth able to compress the noise in few microseconds. ${ }^{8}$ Since the line 1 pulse duration is $\approx 1 \mu$ s that is shorter then the response time of the PLL, we modified the LLRF layout to have a duration of $4 \mu \mathrm{s}$, but reducing the output power for the first $3 \mu \mathrm{s}$. Thus the PLL can act on the first part on the pulse to minimize the jitter while the nominal field level is reached in the last microsecond. ${ }^{9}$ The performance of the klystron stabilization loop has been measured observing the time jitter inside the linac accelerating structures and it results to be $\approx 50 \mathrm{fs}_{R M S}$.

The C-band klystron is presently used for RF conditioning and test of the C-band accelerating structures designed at LNF for the SPARC_LAB injector energy upgrade. ${ }^{7}$ Its modulator is based on solid state HV power supply technology and the phase stability specification (both intra-pulse and pulse-to-pulse) already meets the machine requirements, so that the klystron loop is not necessary.

\subsection{On beam measurements}

The system demonstrated to be very effective in the stability of the accelerator operation. In particular, using a RF compressed beam, we are sensitive to the accelerating field phase inside the accelerating section \#1, that affects the characteristics of the beam (in terms of longitudinal phase space) at the linac end. The jitter of the beam with respect to the RF phase is in fact a combination of the RF phase noise inside the accelerating structure and the PC laser time of arrival on the cathode. To characterize the longitudinal stability of the electron beam we used two independent measurement setups: a RF deflector ${ }^{10}$ and an EOS 
system. ${ }^{11}$ We report about the measurements done on a RF compressed beam, since the EOS setup needs high peak current beams to have a good resolution. In this special acceleration regime, the particles are longitudinally pushed to follow a certain phase of the RF field, reducing the contribution of the PC laser arrival time on the cathode to the total e-bunch time jitter. The RF deflector measurement is performed by observing the vertical jitter of the centroid of the beam on the target. The measurement data take into account the beam jitter with respect to the RF phase noise inside the deflector and are shown in figure $5(\mathrm{a})$. The resulting time jitter is $\approx 90 \mathrm{fs} R M S$. The second method is to observe the jitter of the beam centroid on the time axis projected in the EOS camera. The camera calibration yielded to a sensitivity of $11 \mathrm{fs} /$ pixel. The system uses a replica of the PC laser pulse to sample the longitudinal electron beam shape. The replica is $90 \mathrm{fs}_{R M S}$ long and this limits the resolution for the bunch length measurement, while the resolution on the time of arrival remains unchanged. We measured the relative time jitter between the electron bunch and the laser arrival time at the EOS station. We observed $\approx 80 \mathrm{fs} R M S$ and the acquired data are shown in figure 5(b). These results shows that the system meets the requested specifications with a large margin. Nevertheless, since from the single oscillator measurements we estimated a slightly lower relative jitter between subsystems, we are now investigating whether the beam measurement setups are limited in resolution or there are noise sources we didn't take into account.

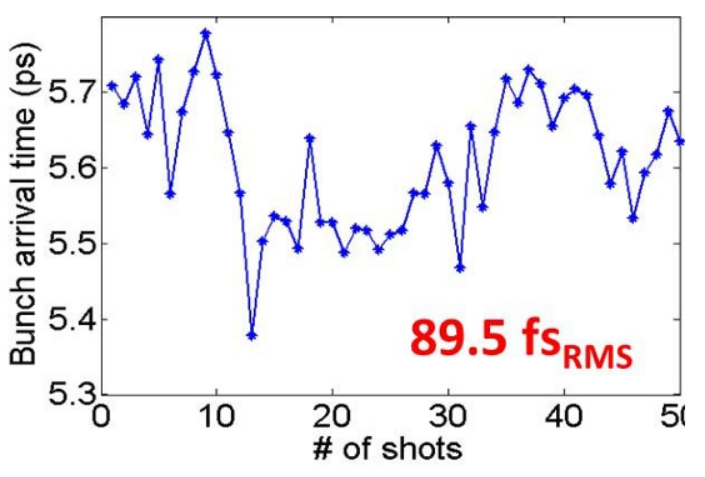

(a)

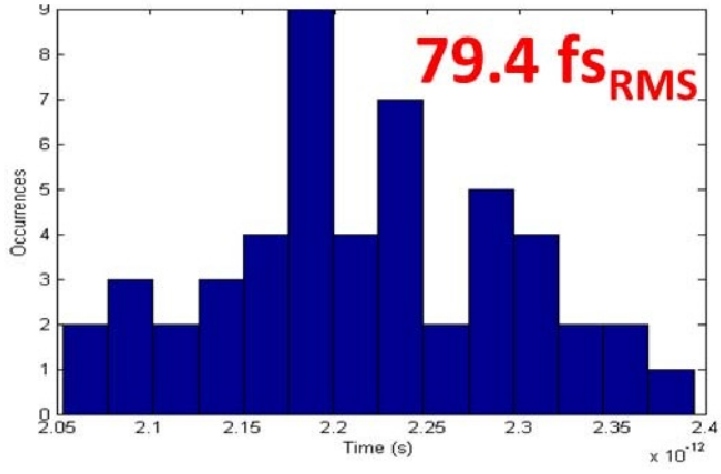

(b)

Figure 5. Diagnostics on the electron beam time jitter

\section{SYSTEM UPGRADE}

A new experiment is planned at the SPARC_LAB facility, involving the creation of a plasma accelerating wave by means of the amplified FLAME laser pulse and the injection of an ultra short electron bunch produced by SPARC for post acceleration. ${ }^{4}$ Simulations yield to a synchronization requirement of $<30$ fs $R M S$ for the relative time of arrival jitter between the two laser and electron pulses. The way to achieve such a stability is well known in the community of large accelerator complexes based on photoinjectors. The synchronization system architecture has to migrate towards the optical reference signal generation and distribution. For a detailed description of the working principles and bibliography references of such systems already implemented in many research institutes see. ${ }^{12}$ We describe in the following paragraphs the SPARC_LAB system upgrade to optical architecture. Figure 6 describes how the synchronization layout changes from electrical to optical.

\subsection{Optical Master Oscillator}

We already purchased and installed the new Optical Master Oscillator (OMO), a pulsed ( $<200 \mathrm{fs}) 1560 \mathrm{~nm}$ fiber laser with $79+1 / 3 \mathrm{MHz}$ repetition rate, that will guarantee a precise reference to the complex. A characterization of such a device is reported in figure 4, where the phase noise spectral density of the oscillator locked to the RMO is reported. In this case the locking to the RMO is needed only to preserve the laser repetition rate, avoiding long term drifts and compressing the low frequency portion of the SSB noise 
spectral density. A new RMO providing an integrated absolute phase noise $<30 f_{\mathcal{S}_{R M S}}$ has been ordered and will be delivered to LNF in few months.

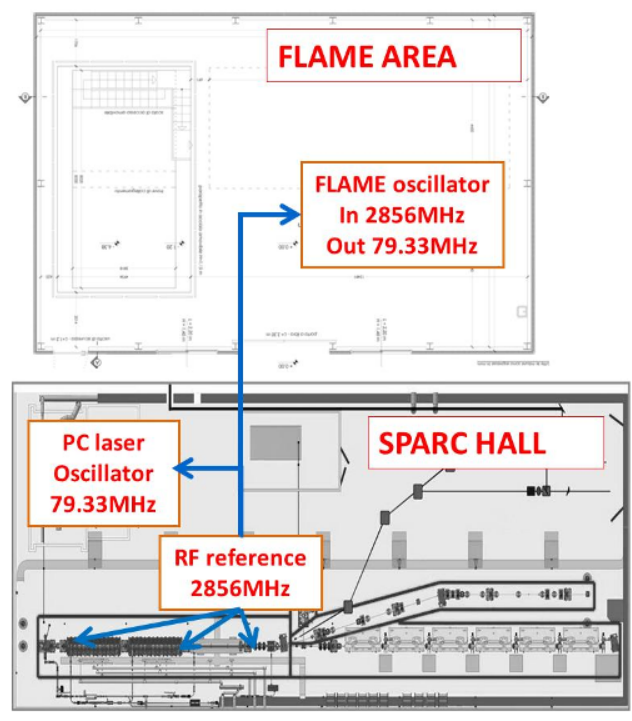

(a)

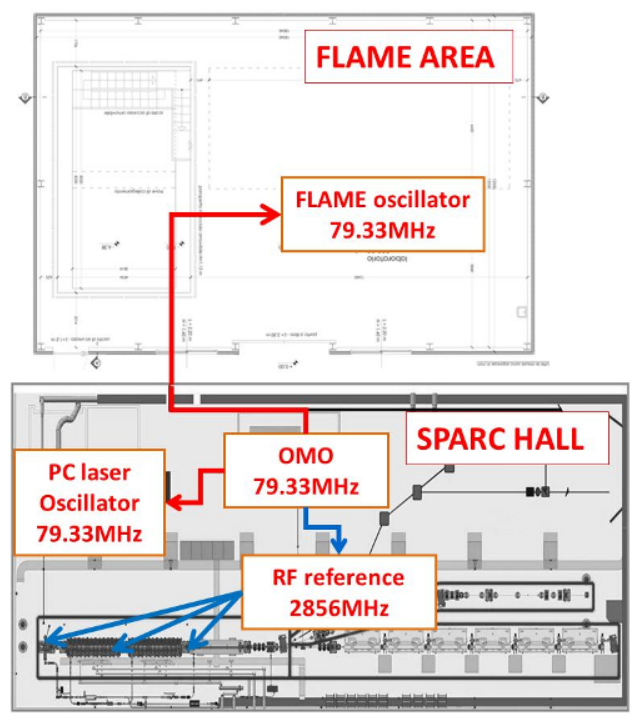

(b)

Figure 6. Layout of the SPARC_LAB synchronization scheme with (a) electrical reference distribution presently used and (b) optical distribution available after the system upgrade.

\subsection{Stabilized fiber links}

Commercial fiber stabilized links are available and they guarantee a 'drift free' reference (few $\mathrm{fs}_{R M S}$ ) at each client location, preserving the very good stability specifications of the OMO. To do so the links need dispersion compensation and active length stabilization. In the SPARC_LAB complex we are about to install one fiber link from the synchronization room located in the SPARC bunker to the FLAME clean room. The total path is $40 \mathrm{~m}$ long.

\subsection{Optical phase detection}

Next step will be to install optical cross-correlators in the laser clean rooms to replace the electronic phase detection scheme with an optical one. The cross-correlators are able to detect the relative time arrival of two laser pulses at $1560 \mathrm{~nm}(\mathrm{OMO})$ and $800 \mathrm{~nm}$ (laser client) with a sensitivity of $10 \mathrm{mV} / \mathrm{fs}$. This will increase the performance of the PLLs around the laser oscillators.

\section{CONCLUSION}

The SPARC_LAB synchronization system has shown so far very good performances and reliability providing the machine stability needed to successfully perform experiments involving short $(<200 \mathrm{fs})$ electron and photon beams. The system has been completely designed and implemented at LNF and has been upgraded many times in the last years. So far the system can guarantee a relative time jitter $<90 \mathrm{fs}_{R M S}$ between the interacting photon and electron pulses. Further development and upgrades are foreseen as described in the paper. In particular next steps will be (i) the design of a new active filter for the stabilization loop of the photocathode laser oscillator, and (ii) the installation of the optical devices in the system (stabilized optical signal distribution and optical phase detectors) that should guarantee a synchronization of $<30 \mathrm{fs}_{R M S}$. The upgraded system is foreseen to be operative by the end of 2015 . 


\section{ACKNOWLEDGMENTS}

This work has been partially funded by the EU Commission in the Seventh Framework Program, Grant Agreement 312453 EuCARD-2 and partially funded by the Italian Minister of Research in the framework of FIRB - Fondo per gli Investimenti della Ricerca di Base, Project n. RBFR12NK5K.

\section{REFERENCES}

[1] M. Ferrario et al., Nuclear Instruments and Methods in Physics Research Section B: Beam Interactions with Materials and Atoms, Volume 309, 15 August 2013, Pages 183188

[2] C. Vaccarezza et al., Proceedings of IPAC2014, Dresden, Germany, MOPRO078

[3] M. Ferrario et al., Nuclear Instruments and Methods in Physics Research A 637 (2011) S43S46

[4] A. R. Rossi et al., Proceedings of IPAC2012, New Orleans, Louisiana, USA, WEEPPB002

[5] M.Bellaveglia and A. Gallo, SPARC-RF-14/002, LNF technical note, http://www.lnf.infn.it/acceleratori/sparc/technotes.html

[6] A. J. Fleming, 2010 American Control Conference Marriott Waterfront, Baltimore, MD, USA June 30-July 02, 2010, FrA08.4

[7] D. Alesini et al., Proceedings of IPAC2014, Dresden, Germany, MOOCA01

[8] M. Bellaveglia, A. Gallo, C. Vicario, in EUROFEL-Report-2006-DS3-027

[9] M. Bellaveglia et al., SPARC-RF-12/001, LNF technical note, http://www.lnf.infn.it/acceleratori/sparc/technotes.html

[10] M. Bellaveglia, et al., in Proceedings of DIPAC09, TUPD30

[11] R. Pompili, in PhD Thesis 2013, www.infn.it/thesis/thesis_dettaglio.php?tid $\overline{8} 140$

[12] M. Bellaveglia, IL NUOVO CIMENTO, Vol. 37 C, N. 4, DOI 10.1393/ncc/i2014-11815-2 